\title{
A Dilemma on Re-biopsy in the Era of Precision Cancer Therapy
}

\author{
Yuichi Takiguchi \\ Key words: ALK fusion gene, immunohistochemistry, fluorescence in situ hybridization, chemotherapy, \\ transient alteration, non-small cell lung cancer
}

(Intern Med 55: 1683-1684, 2016)

(DOI: 10.2169/internalmedicine.55.6567)

Molecular targeted therapy and immune-checkpoint therapy have evolutionally changed cancer drug therapy. Consequently, the survival of patients with cancer harboring a molecular target has dramatically improved. Tissue sampling for analyzing the molecular targets before starting therapy is the bottom line in an era of personalized or precision medicine. For example, the first step of the algorithm for decision-making in treating patients with non-small cell lung cancer (NSCLC) is to detect either an epidermal growth factor receptor $(E G F R)$ or anaplastic lymphoma kinase $(A L K)$ mutation (1). This step is not so difficult because the mutations are examined using tissue samples for the normal pathological diagnosis of cancer. Recent advancements in molecular targeted therapy for NSCLC, however, are now demanding more precise analyses.

Patients with NSCLC harboring the EGFR mutation mostly enjoy a long progression free survival-time while being treated with an EGFR-tyrosine kinase inhibitor (TKI). Nevertheless, the cancer normally becomes resistant to EGFR-TKIs within a couple of years. The secondary mutation of T790M on EGFR in $49 \%$ and the transformation to small cell lung carcinoma (SCLC) in $14 \%$ constitute a part of this resistance mechanism (2). New generation EGFRTKIs which are specifically effective for T790M mutated EGFR are expected to become available soon. Diagnosing SCLC transformation is also clinically important because the disease is sensitive for specific chemotherapy. The resistance mechanism to ALK-TKIs includes an acquired mutation in EGFR (3) that is sensitive to EGFR-TKIs. In addition, an evolutional development of immune-checkpoint therapy for cancer has prompted the possible need to evaluate the PDL1 expression at the time of cancer recurrence in patients with NSCLC (4). Therefore, when NSCLC with an EGFR or $A L K$ mutation becomes resistant to an EGFR-TKI or an ALK-TKI, respectively, or possibly when any NSCLC be- comes resistant to any first-line chemotherapy, then tissue sampling to elucidate the resistance mechanism and evaluate the PD-L1 expression at the time of tumor recurrence by means of a re-biopsy is required.

The re-biopsy, however, in patients with advanced and relapsed lung cancer is often technically difficult to perform, and it is also associated with some significant risk in such patients. Although a re-biopsy is still not a common procedure in the current practice, physicians treating lung cancer are required to overcome the problem of re-biopsy before the new generation EGFR-TKIs will be approved.

An important case report published in this issue of Internal Medicine raised an additional issue associated with rebiopsy. Taniguchi et al. reported a patient who had lung adenocarcinoma with an $A L K$ translocation (5). The patient was treated with chemoradiotherapy followed by consolidation chemotherapy, and thereafter the cancer was surgically resected. Nevertheless, the cancer unfortunately recurred at 15 months after surgery, and the recurred cancer was rebiopsied to clarify the type of recurrence. ALK expression assessed by immunohistochemistry (IHC) was positive at the first diagnosis of cancer and at the recurrence after surgery, but it was negative at the time of surgery, that is, at the time chemoradiotherapy was completed, while it was fluorescence in situ hybridization (FISH) positive for $A L K$ translocation throughout the entire clinical course. Such a conditional alteration in the mutated ALK expression according to cytotoxic chemotherapy sounds reasonable, whereas this new finding should be seen as a warning to physicians who are responsible for diagnosing the molecular targets of cancer. Currently, $A L K$ translocation is diagnosed by any of three methods, IHC, FISH or reverse-transcriptase polymerase chain reactions, with the first two methods being covered by the Japanese health insurance system. The guidelines of the Japanese Lung Cancer Society place IHC testing 
above FISH testing, because of the technical difficulties and high cost of FISH (6). As shown by Taniguchi et al., if IHC testing could be performed at the completion of cytotoxic chemotherapy, an algorithm prioritizing IHC over FISH would fail to detect the $A L K$ fusion gene. An acquired decrease in HER2 expression in breast cancer due to chemotherapy is also demonstrated in preclinical and clinical situations (7-9). PD-L1 expression in cancer is also considered to be a dynamic marker. It is therefore necessary to elucidate the optimal timing and the best methods for performing re-biopsy to achieve the best therapeutic outcome and to lower the patients' burden. While the goal of precision medicine is to achieve a better outcome, it increases the burden on patients since it requires that they undergo painful re-biopsies, while also increasing the stress on physicians due to the need to perform sophisticated re-biopsies.

\section{Author's disclosure of potential Conflicts of Interest (COI).}

Yuichi Takiguchi: Honoraria, Ono pharmaceutical.

\section{References}

1. Masters GA, Temin S, Azzoli CG, et al. Systemic therapy for stage IV non-small-cell lung cancer: American Society of Clinical Oncology Clinical Practice Guideline Update. J Clin Oncol 33: 3488-3515, 2015.
2. Sequist LV, Waltman BA, Dias-Santagata D, et al. Genotypic and histological evolution of lung cancers acquiring resistance to EGFR inhibitors. Sci Transl Med 3: 75ra26, 2011.

3. Doebele RC, Pilling AB, Aisner DL, et al. Mechanisms of resistance to crizotinib in patients with ALK gene rearranged nonsmall cell lung cancer. Clin Cancer Res 18: 1472-1482, 2012.

4. Naiyer AR, Edward BG, Natasha L, et al. Optimizing PD-L1 as a biomarker of response with pembrolizumab (pembro; MK-3475) as first-line therapy for PD-L1-positive metastatic non-small cell lung cancer (NSCLC): Updated data from KEYNOTE-001. J Clin Oncol 33 (Suppl): abstr 8026, 2015.

5. Taniguchi H, Ikeda $\mathrm{T}$, Soda $\mathrm{H}$, et al. Changes in immunohistochemical protein levels in anaplastic lymphoma kinase-positive lung adenocarcinoma possibly due to chemo-radiotherapy. Intern Med 55: 1775-1778, 2016.

6. Yatabe Y, Satouchi M, Akita H, et al. Guidance for ALK fusion gene testing in patients with lung cancer (in Japanese) [Internet]. [cited 2016 Apr. 29]. Available from: https://www.haigan.gr.jp/upl oads/photos/1039.pdf

7. Kumaki N, Umemura S, Tang X, Saito Y, Suzuki Y, Tokuda Y. Alteration of immunohistochemical biomarkers between pre- and post-chemotherapy: hormone receptors, HER2 and Ki-67. Breast Cancer 18: 98-102, 2011.

8. Li P, Liu T, Wang Y, et al. Influence of neoadjuvant chemotherapy on HER2/neu status in invasive breast cancer. Clin Breast Cancer 13: 53-60, 2013.

9. Goltsov A, Deeni Y, Khalil HS, et al. Systems analysis of druginduced receptor tyrosine kinase reprogramming following targeted mono- and combination anti-cancer therapy. Cells 3: 563591, 2014.

(C) 2016 The Japanese Society of Internal Medicine http://www.naika.or.jp/imonline/index.html 